\title{
Modelo de Acción Docente como Alternativa Pedagógica para el Manejo del Trastorno Disocial Escolar
}

\author{
Jesús A. Gómez \\ Universidad de Otavalo, Carrera de Derecho. Av, Sarances y los Pendoneros, Otavalo, Ecuador. \\ (e-mail: jgomez@uotavalo.edu.ec jesgomez35@gmail.com)
}

Recibido Jun. 19, 2017; Aceptado Ago. 24, 2017; Versión final Oct. 4, 2017, Publicado Abr. 2018

\begin{abstract}
Resumen
Se propone un modelo de acción docente desde la perspectiva humanista como alternativa pedagógica para el manejo del trastorno disocial escolar. El estudio está dirigido a los docentes de Educación Secundaria de la Unidad Educativa Alto Barinas Norte en Venezuela. Este tipo de trastorno disocial, se caracteriza por comportamientos violentos, agresivos que infringen las normas de convivencias. La metodología utilizada fue de tipo proyectivo que llevó el estudio a generar el modelo. Se utilizó como instrumento de recolección de datos, el registro de campo y el cuestionario aplicado a los 19 docentes de la muestra del estudio. Como resultado, se describió los tipos de comportamiento relacionados con el trastorno disocial y se identificó en el docente la falta de competencias para manejar este trastorno. En conclusión, se diseñó un buen modelo de acción docente para fortalecer los tipos de competencias básica, profesional y participativa, a fin de prevenir el trastorno disocial escolar.
\end{abstract}

Palabras clave: modelo educacional; acción docente; alternativa pedagógica; trastorno disocial escolar

\section{Teaching Action Model as a Pedagogical Alternative for the Management of School Dissocial Disorder}

\begin{abstract}
A model of educational action from the humanist perspective is proposed as a pedagogical alternative for the management of the disocial disorder addressed to secondary education teachers of the Educational Unit Alto Barinas Norte in Venezuela. This type of dissocial disorder is characterized by violent, aggressive behavior that violates the norms of coexistence. The methodology used was of a projective type that led the study to generate the model. The field record and the questionnaire applied to the 19 teachers of the study sample were used as data collection instrument. As a result, the types of behavior related to the disorder are described and the lack of skills of the teacher to deal with this disorder was identified. In conclusion, a good model of teaching action was designed to strengthen the types of basic, professional and participatory competences, in order to prevent school disocial disorder.
\end{abstract}




\section{INTRODUCCIÓN}

Desde décadas atrás, se investigan los problemas de conducta en adolescentes, especialmente los comportamientos agresivos, delictivos, violentos en jóvenes entre 12-16 años, tanto en el ámbito familiar como en el escolar, en base a la percepción de padres y profesores. En el caso de Venezuela, los planteles educativos de Educación Secundaria, enfrentan cambios económicos, políticos, culturales que afectan el comportamiento estudiantil y el desenvolvimiento académico de la institución. Estos cambios se extienden hasta provocar en el estudiante, un déficit de valores, la aparición de nuevos comportamientos, agresividad, el irrespeto a las normas y la violación a los derechos humanos cuyas experiencias personales afectan la conducta (Acevedo et al., 2015). Uno de estos problemas, según (Urazán et al., 2013) es el trastorno disocial, es uno de los más comunes entre los adolescentes que afecta la facultad cognitiva para lograr el aprendizaje. A nivel de educación secundaria, este tipo de trastorno se manifiesta en el joven estudiante mediante un comportamiento caracterizado por la realización repetitiva de actos en contra de las normas sociales, los derechos de los demás, agresiones a personas, destrucción, robo y violación a las normas del grupo de referencia, (Peña y Palacios, 2011).

Por consiguiente, (Vera et al., 2013) expresa a través de un estudio de tesis doctoral "Conducta Antisocial: Asociación con Psicopatología en Niños y Adolescentes" con el propósito de estudiar la prevalencia y la asociación diferencial de las conductas antisociales con una mayor gravedad de los problemas de salud mental, según el sexo y la edad de los individuos. Concluyó que las conductas antisociales en adolescentes, son altamente prevalentes, constituyen una de las principales causas de consultas recurrentes a centros de salud mental, generan diversas dificultades sobre el funcionamiento cotidiano de los individuos y su entorno social, El estudio se fundamentó bajo el paradigma cualitativo, los métodos utilizados fueron modelos de regresión logística, binomial-negativa, múltiple, y con modelos de ecuaciones estructurales.

Seguidamente, los resultados de este estudio permitieron detectar la presencia de conductas antisociales en los jóvenes en edades comprendidas entre los 13 y 17 años de edad, la cual estaba asociada mayormente a problemas relacionados con el sexo y la sobreprotección por parte de los padres en quienes aceptaban en ellos este tipo de comportamiento. La presencia de conductas antisociales no difirió significativamente según el sexo o la edad de los individuos, no obstante, se observó una prevalencia mayor de estas conductas entre los 13 y los 17 años, y en el sexo masculino, estableció como fundamental la valoración de las conductas antisociales en escolares cuyas edades oscilen entre dichos límites. Por ello, en la Unidad Educativa "Alto Barinas Norte" ubicada en la ciudad de Barinas, parroquia Alto Barinas, municipio Barinas del estado Barinas, Venezuela, se observó situaciones individuales y grupales similares a este hecho, vinculados con este tipo de trastorno. Tal es el caso, la presencia de agresividad entre los estudiantes de secundaria, la cual en algunas ocasiones involucraron a miembros de otros niveles de la institución, poniéndose de manifiesto amenazas, agresiones físicas y el incumplimiento a las normas de convivencia escolar. Considerando las definiciones dadas de trastorno disocial, tales expresiones conductuales fueron asumidas como síntomas o probables manifestaciones de este.

Una realidad científica que otorga importancia al atender en forma sistematizada, la ocurrencia de amenazas, agresiones físicas e incumplimiento a las normas de convivencia escolar desde el aula, es la correlación clínica, entre las conductas que definen el trastorno disocial con el consumo de alcohol y drogas por adolescentes, confirmada por (Peña y Palacios, 2011), quien señala que más del $60 \%$ de los adolescentes con trastorno disocial tienen algún tipo de abuso o dependencia al alcohol o drogas, conclusión ratificada más tarde (Pozo y González 2009) al afirmar que cuando las conductas asociadas con trastorno disocial presentes en la adolescencia continúan en la adultez, son problemas que suelen continuar en forma de conducta criminal, alcoholismo, afectación psiquiátrica grave, dificultades de adaptación manifiestas en el trabajo y la familia y problemas interpersonales.

De igual manera, se evidenció en el docente, confusión para identificar el trastorno disocial en el ambiente escolar, caracterizados por comportamientos inadecuados que perjudican en el bienestar del estudiante. De allí, (Ornelas et al., 2012), la institución educativa, no cuenta con una herramienta pedagógica que sirva de apoyo al docente para abordar este trastorno y sus comportamientos; Emplear las estrategias adecuadas, conceptos teóricos, que a su vez permita la prevención y diferenciar en el estudiante este tipo de conducta no deseada. Por ejemplo, el docente a través de su rol de orientador, puede guiar el comportamiento del estudiante, bríndale la ayuda necesaria, la asesoría, con el propósito de contribuir con el desarrollo personal y académico. En consecuencia, el presente artículo, tiene como finalidad dar respuesta al siguiente problema científico, ¿Cómo contribuir con el fortalecimiento de la acción docente para el manejo del trastorno disocial escolar?

Desde este punto de vista, puede reiterarse que la aparición de este tipo de comportamiento en la personalidad de estudiante, puede prevenirse mediante la acción docente, es decir la actividad orientadora, 
que impulsa la autoestima, el crecimiento y el desarrollo saludables. Como también, conocer estrategias de intervención para orientar al joven estudiante, potenciar la toma de decisiones, promover la participación en el proceso de enseñanza, generar comportamientos saludables y actitudes para un mejor desempeño académico dentro del ambiente escolar, (Aznar y Laiton, 2017).

La acción pedagógica, (Rosa et al., 2015), está caracterizada por promover en el estudiante situaciones significativas que contribuyan con su formación integral. Así que, a través de su accionar, el docente orienta el comportamiento del estudiante, hacia el logro de los objetivos de aprendizaje, el cumplimiento de las normas de convivencia escolar y el reconocimiento de la importancia que tienen los valores humanistas para la vida. Además de ello, proporciona al profesor, desarrollar habilidades cognitivas, competencias personales, así como estrategias de gestión de las emociones para actuar conforme a la realidad, (Flores et al., 2012). La teoría de la acción docente de Talcott Parsons, (Almeida, 2001; Contreras, 2009), es un enfoque que orienta la acción pedagógica como un hecho humano visible, tangible, sistemático, ejercido por el docente con el objetivo primordial de llevar a cabo un proceso de interacción de enseñanza aprendizaje a fin de lograr la formación integral del educando.

Por consiguiente (Bontempi y Pietri, 2017) han demostrado que la acción docente tiene como responsabilidad promover experiencias de aprendizaje, desarrollar tareas, tomar decisiones que contribuyan en el logro de las metas del estudiante, la construcción del conocimiento, habilidades que faciliten el manejo de la conducta, especialmente de aquellas relacionadas o proclives a comportamientos disóciales, a partir de la disminución de la agresividad y la mejora de la armonía en el plantel educativo, mediante el cumplimiento de las normas de convivencia escolar. Aunado a este aspecto, (Vieira y Pinheiro, 2013), expresan que la teoría de Carl Rogers, se fundamenta en el enfoque centrado en la persona, las relaciones humanas, basado en lo que el determinó como la persona plenamente funcional, la conducta propia, valora la armonía entre el hombre y el yo. Al igual, (Dutra et al., 2016), declaran esta teoría se fundamenta en la Psicología Humanista que asume como preferencia la experiencia, la vivencia, el comportamiento y la subjetividad de la persona según su mundo interior, como también, estados anímicos, valores, relaciones interpersonales y el autoconcepto.

En tal sentido, (Bryan et al., 2015), aseveran que el enfoque centrado en la persona de Carl Rogers, el profesional es un educador que puede influenciar en el comportamiento del estudiante dentro de la institución mediante la formación de las relaciones humanas. De ahí, (Zalyaeva y Solodkova, 2014), ratifican el docente utiliza distintos roles que se adecuan a las necesidades del joven estudiante, así como en situaciones específicas que desempeña una función clave el procesos de enseñanza aprendizaje. Siguiendo este planteamiento, (Todorescu et al., 2015; Blašková et al., 2014), aseguran, el docente puede ejercer el rol de orientador en el ambiente escolar, cuya autoridad intelectual permite aconsejar, asesorar el comportamiento estudiantil, como agente de cambio, líder y facilitador que brinda apoyo en la institución educativa. De lo antes descrito, el docente en su rol como orientador, se apoya en la capacidad propia de cada individuo para fortalecer las relaciones interpersonales, fomentar el cumplimiento de las normas de convivencia y despierta el interés en el estudiante por los valores humanos como lo es el respeto a los demás como personas.

Por tanto, (Carvalho y Novo, 2014), confirman la manera como el estudiante expresa su convivencia, actuación, proceder, comportamiento escolar en la institución amerita implantar un programa o modelo que ayude al docente establecer estrategias que faciliten al estudiante una adecuada orientación individual y una buena conducta. De allí, la relación que existe entre la teoría de Carl Rogers y el rol del docente como orientador desempeña un papel clave en la vida del adolescente, ayuda al mismo a ser electivo, capaz de adoptar su propio comportamiento para lograr metas de vida, a ser responsable en las decisiones, actos volitivos que reflejan en su correcta acción. En consecuencia, un modelo de acción docente fundamentado en la perspectiva humanista, centrado en el estudiante, representa según (Aarón, et al., 2016), un esquema epistémico referido a conceptos teóricos, estrategias, relacionados con hechos reales que proporcione respuesta a la situación problemática abordada. En efecto, (Cid, et al., 2017), el modelo se fundamenta en la teoría centrada en la persona de Carl Roger y la acción docente desde la teoría de Talcott Parsons, a fin de proporcionar elementos clave para fortalecer la gestión docente desde el aspecto humanista, mediante talleres para la formación en competencias que faciliten el manejo del trastorno disocial escolar en los estudiantes de secundaria.

Por tanto, el presente estudio abordará las siguientes preguntas de investigación: ¿Qué tipo de comportamientos disociales existen en los estudiantes de educación secundaria en la institución objeto de estudio? ¿Cuáles competencias poseen los docentes de la Unidad Educativa Alto Barinas, para manejar el trastorno disocial escolar en los estudiantes de educación secundaria? ¿Qué tipo de competencias debe poseer el docente para manejar el trastorno disocial escolar en los estudiantes de educación secundaria? $Y$ por último, ¿Qué características y elementos deben componer un modelo de acción docente para ser una estrategia pedagógica dirigida al manejo del trastorno disocial escolar en los estudiantes de secundaria del plantel en cuestión? 


\section{Objetivos de la Investigación}

Se presentan el objetivo general y cuatro objetivos específicos, a saber:

\section{Objetivo General}

Generar un modelo de acción docente desde la perspectiva humanista, como alternativa pedagógica para el manejo del trastorno disocial escolar dirigido a los docentes de educación secundaria de la Unidad Educativa "Alto Barinas Norte", ubicada en la parroquia Alto Barinas, municipio Barinas estado Barinas, Venezuela.

\section{Objetivos Específicos}

Describir los tipos de comportamientos disóciales existentes en los estudiantes de educación secundaria en la Unidad Educativa "Alto Barinas Norte", ubicada en la parroquia Alto Barinas municipio Barinas estado Barinas, Venezuela.

Identificar las competencias en el docente para el manejo del trastorno disocial escolar en los estudiantes de educación secundaria.

Establecer el tipo de competencia en el docentes para el manejo del trastorno disocial escolar en los estudiantes de educación secundaria

Diseñar un modelo de acción docente desde la perspectiva humanista como alternativa pedagógica para el manejo del trastorno disocial escolar.

Razón por la cual, (Vieira y Pinheiro, 2013), concibe la perspectiva humanista como el estudio centrado en la persona de la teoría de Carl Rogers, así como también el comportamiento, las relaciones interpersonales que garantizan la armonía entre el individuo y su ser interior. Por tanto, (Pérez, 2011; Contreras, 2009) expresan el modelo de acciones docente propuesto, se enfoca en el humanismo el cual facilita la interacción entre el orientador y el orientado, el docente como orientador promueve un proceso de ayuda, asesoría, consejería y un ambiente de interacción positiva para generar un buen comportamiento en el estudiante. En efecto, este modelo surge como una alternativa del modelo pedagógico psicométrico existente en la institución educativa, considerado rígido, tradicionalista, limitado para formular datos y propiciar la ayuda necesaria.

\section{MATERIALES Y MÉTODOS}

A continuación, se presenta la metodología empleada (investigación proyectiva), la muestra (muestreo probabilístico o aleatorio) y los instrumentos utilizados (recolección de datos)

\section{Metodología}

La metodología empleada en el estudio fue investigación proyectiva, (Barrera, 2007) expresa que este tipo de metodología consiste en explorar, describir, explicar y proponer mediante el diseño de una propuesta que responda a la necesidad de una realidad o problemática. El término proyectivo es una aproximación, un modelo teórico soportado en una teoría, procesos y estrategias propias que permitió generar un modelo de acción docente para el manejo del trastorno disocial escolar en los estudiantes de la Unidad Educativa "Alto Barinas Norte"

\section{Muestra}

El tipo de muestreo escogido para la selección de la muestra fue de tipo probabilístico o aleatorio, esta posee menos sesgo que las técnicas de muestreo no probabilísticas, (Hernández et al., 2014). La selección de la muestra consistió en seleccionar los integrantes de acuerdo con un procedimiento sencillo, se depositó en un recipiente un número de papeletas correspondiente al total de docentes de aula, cada una contentiva del nombre de uno; luego se procedió a extraer el 30\% lo cual representó el equivalente a diecinueve (19) papeletas.

\section{Instrumentos}

Los instrumentos de recolección de datos, representan la herramienta para la recopilación de la información sobre el objeto de estudio (Bastos et al., 2014). Entonces, se utilizó el registro de campo con el propósito de recoger hechos, incidentes, situaciones de manera objetiva para describir la convivencia en el ambiente escolar, obtenidas estas evidencias se hace una síntesis de estos datos para construir un informe, (Lapponi 
et al., 2016). En efecto, este fue utilizado por el investigador para registrar los tipos de comportamiento como se evidencia en el resultado de la Tabla 1: Clasificación de los comportamientos observados en la Unidad Educativa "Alto Barinas Norte".

Así como también, se utilizó como instrumento el cuestionario (Faleiros et al., 2016) han demostrado que representa una estrategia para la recolección de datos en un estudio. Esta herramienta estuvo estructurada en 22 ítems, con opción de repuesta Si o No, lo cual permitió obtener la información necesaria directamente de las opiniones de los 19 docentes de la institución educativa. Los resultados de este cuestionario fueron evidenciados posteriormente en la Tabla 3: Distribución de la opinión de los docentes con relación a la función del rol orientador. La validez del cuestionario se fundamentó en la técnica de juicio de experto, (García y Suárez 2013; Jorm, 2015), señalan que es una técnica que garantiza la pertinencia, redacción y adecuación entre los ítems del instrumento mediante el consenso entre expertos, investigadores que facilitaron la obtención de resultados confiables. De igual manera, la confiabilidad del cuestionario se determinó a través del coeficiente de Kuder Richarson, KR20, (Cascaes et al. 2015), este medio determinó la consistencia interna y la corresponsabilidad de los ítems con los objetivos de la investigación.

\section{RESULTADOS Y DISCUSIÓN}

El presente apartado está referido a los datos obtenidos de los instrumentos aplicados en el estudio con la finalidad de establecer conclusiones pertinentes, (Hernández, et al., 2014). La interpretación de los resultados, consistió en la inferencia directa sobre los datos obtenidos de cada instrumento, las teorías y los objetivos con el propósito de constituir las conclusiones de la investigación. En cuanto al primer instrumento registro de campo utilizado por el investigador, este permitió describir los tipos de comportamiento disóciales en los estudiantes de educación secundaria como se observa en la Tabla 2.

Tabla 1: Clasificación de los comportamientos observados en la Unidad Educativa "Alto Barinas Norte".

\begin{tabular}{|c|c|c|c|}
\hline Comportamiento observado & Frecuencia & Clasificación & Acción docente asumida \\
\hline $\begin{array}{l}\text { En el laboratorio de informática, un estudiante } \\
\text { amenaza a otro vía Skipe, con agredirlo si lo delata } \\
\text { ante la Dirección o sus padres }\end{array}$ & 1 & $\begin{array}{l}\text { Comportamiento } \\
\text { agresivo }\end{array}$ & $\begin{array}{l}\text { El docente del aula observó el hecho sin } \\
\text { tomar acción alguna. }\end{array}$ \\
\hline $\begin{array}{l}\text { En la cancha un estudiante de forma directa y actitud } \\
\text { amenazante le advierte a otro que se cuide }\end{array}$ & 1 & $\begin{array}{l}\text { Comportamiento } \\
\text { agresivo }\end{array}$ & $\begin{array}{l}\text { El docente se limitó a preguntar qué } \\
\text { pasaba }\end{array}$ \\
\hline $\begin{array}{l}\text { En el aula, un estudiante simulando descuido impacta } \\
\text { a otro que lo acusó de haberse copiado en el examen }\end{array}$ & 2 & $\begin{array}{l}\text { Comportamiento } \\
\text { agresivo }\end{array}$ & $\begin{array}{l}\text { El docente se limitó a recomendar que } \\
\text { tuviesen cuidado al caminar }\end{array}$ \\
\hline $\begin{array}{l}\text { Un estudiante golpea a otro ocasionándole lesiones } \\
\text { graves en el cuerpo }\end{array}$ & 5 & $\begin{array}{l}\text { Comportamiento } \\
\text { agresivo }\end{array}$ & $\begin{array}{l}\text { El docente del aula observó el hecho sin } \\
\text { tomar acción alguna. }\end{array}$ \\
\hline $\begin{array}{l}\text { Un estudiante al momento de entrar por la puerta } \\
\text { principal de la institución, golpeó al portero, quien le } \\
\text { llamó la atención }\end{array}$ & 1 & $\begin{array}{l}\text { Comportamiento } \\
\text { agresivo }\end{array}$ & $\begin{array}{l}\text { El portero notificó el hecho ante la } \\
\text { Dirección y el Docente Guía reprochó la } \\
\text { actitud al estudiante }\end{array}$ \\
\hline $\begin{array}{l}\text { Grupo de estudiante acuerda ocultarle los cuadernos } \\
\text { a un compañero para burlarse de él }\end{array}$ & 18 & $\begin{array}{l}\text { Incumplimiento a las } \\
\text { normas de } \\
\text { convivencia escolar }\end{array}$ & $\begin{array}{l}\text { Ante la presencia del docente, los } \\
\text { estudiantes se dispersan. Nadie reconoce } \\
\text { falta }\end{array}$ \\
\hline $\begin{array}{l}\text { Un estudiante arrebata cuadernos a un compañero, } \\
\text { los arroja al piso y patea }\end{array}$ & 4 & $\begin{array}{l}\text { Comportamiento } \\
\text { agresivo }\end{array}$ & $\begin{array}{l}\text { Ante la presencia del docente, los } \\
\text { estudiantes se dispersan. El agredido es } \\
\text { consolado }\end{array}$ \\
\hline $\begin{array}{l}\text { Estudiantes usan términos y frases, despectivos o } \\
\text { soeces, para llamar a sus compañeros }\end{array}$ & 35 & $\begin{array}{l}\text { Incumplimiento a las } \\
\text { normas de } \\
\text { convivencia escolar }\end{array}$ & $\begin{array}{l}\text { El docente llama la atención sobre el uso } \\
\text { de las palabras cuando son soeces, de lo } \\
\text { contrario hace caso omiso }\end{array}$ \\
\hline $\begin{array}{l}\text { Un estudiante amenaza a otro con usar una pistola } \\
\text { por diferencias personales, en presencia de otros } \\
\text { estudiantes }\end{array}$ & 2 & $\begin{array}{l}\text { Comportamiento } \\
\text { delictivo, }\end{array}$ & $\begin{array}{l}\text { El docente expresó desconocer qué } \\
\text { hacer }\end{array}$ \\
\hline $\begin{array}{l}\text { Un estudiante amenaza a otro con usar una navaja, } \\
\text { por celos }\end{array}$ & 1 & $\begin{array}{l}\text { Comportamiento } \\
\text { delictivo, }\end{array}$ & $\begin{array}{l}\text { El docente llama la atención sobre el uso } \\
\text { de las armas y la resolución de conflictos } \\
\text { mediante el diálogo }\end{array}$ \\
\hline Sustracción de dos equipos portátiles de computación & 1 & $\begin{array}{l}\text { Comportamiento } \\
\text { delictivo }\end{array}$ & $\begin{array}{l}\text { El docente notificó a la Dirección y se } \\
\text { comunicó con los padres }\end{array}$ \\
\hline Ataques físicos por desacuerdo & 16 & $\begin{array}{l}\text { Comportamiento } \\
\text { agresivo }\end{array}$ & $\begin{array}{l}\text { El docente ignora por ser problemas de } \\
\text { muchacho }\end{array}$ \\
\hline Utilizar la camisa por fuera & 62 & $\begin{array}{l}\text { Incumplimiento a las } \\
\text { normas de } \\
\text { convivencia escolar }\end{array}$ & $\begin{array}{l}\text { El docente llama la atención sobre el } \\
\text { cumplimiento del uniforme y le exige } \\
\text { meterse la camisa }\end{array}$ \\
\hline
\end{tabular}


Tabla 2: Comportamientos disóciales observados en la Unidad Educativa "Alto Barinas Norte".

\begin{tabular}{|l|c|c|}
\hline \multicolumn{1}{|c|}{ Comportamiento Disocial } & F & $\%$ \\
\hline Comportamiento agresivo & 30 & 20 \\
\hline Comportamiento delictivo & 4 & 3 \\
\hline Incumplimiento de las normas de convivencia escolar & 115 & 77 \\
\hline Total & 149 & 100 \\
\hline
\end{tabular}

De esta manera, se logró constatar en la Tabla 2. Comportamientos disociales observados en la Unidad Educativa "Alto Barinas Norte", muestra la incidencia de cada tipo de comportamiento disocial, de acuerdo con la clasificación el comportamiento más común según (Lapponi et al., 2016). Al respecto, un (77\%) está relacionado con el incumplimiento de las normas de convivencia, incluye las inasistencias injustificadas, hacer bromas pesadas, travesuras, irrespeto a otros y conflictividad. Vale acotar, este cuadro no recoge lo relacionado con las inasistencias injustificadas ni los gritos en las conversaciones, lo cual tenía un alto porcentaje de ocurrencia, pero al haber el acuerdo tácito entre los miembros de la comunidad escolar de que son faltas a la normativa, se procedía a acciones mecánicas como registrarlo en el diario y llamar la atención sobre el respeto a las opiniones. En consecuencia, estos resultados provenientes del registro de campo, permitieron el cumplimiento del primer objetivo específico relacionado con la descripción de los tipos de comportamientos disóciales existentes en los estudiantes de educación secundaria de la institución educativa.

En correlación al segundo instrumento el cuestionario, se aplicó a los 19 docentes que conformó la muestra del estudio con el propósito de identificar las competencias docentes para el manejo del trastorno disocial escolar en los estudiantes de educación secundaria en la institución educativa. Se estructuró 22 ítems, con repuesta Si o No. En el primer y segundo ítems del instrumento se tomó como variable; La acción docente, dimensiones; Roles, competencias e impactos; Indicadores; Roles, competencias básicas, profesionales como se evidencia en la Tabla 3:

Tabla 3: Distribución de la opinión de los docentes con relación a la función del rol orientador.

\begin{tabular}{|c|c|c|c|c|c|}
\hline \multirow{2}{*}{$\mathrm{N}^{\circ}$} & \multirow{2}{*}{ Identificación del Rol } & \multicolumn{2}{|c|}{$\mathrm{Si}$} & \multicolumn{2}{|c|}{ No } \\
\hline & & $\mathrm{F}$ & $\%$ & $\mathrm{~F}$ & $\%$ \\
\hline 1 & Como docente, ¿Conoces las funciones del rol de orientador? & 10 & 53 & 9 & 47 \\
\hline 2 & $\begin{array}{l}\text { ¿Aplicas el rol de orientador para ayudar al estudiante con problemas de } \\
\text { comportamientos disocial? }\end{array}$ & 4 & 21 & 15 & 79 \\
\hline
\end{tabular}

En referencia a la Tabla 3, Distribución de la opinión de los docentes con relación a la función del rol orientador, se logró observar en el ítem 1, el cincuenta y tres por ciento (53\%) de los docentes, manifestó si conocen la función del rol orientador, cuarenta y siete por ciento $(47 \%)$ expresaron No. A partir de esta información, existe un grupo de docente que no poseen los conocimientos básicos sobre la función del rol de orientador. El ítem 2, reflejó veintiuno por ciento $(21 \%)$ de los docentes afirman aplicar el rol de orientador para ayudar al estudiante con problemas de comportamientos disocial, un setenta y nueve por cierto (79\%) manifestó No. Por ello, existe una mayoría porcentual de los docentes, no aplican el rol de orientador para ayudar a los estudiantes con problemas de comportamiento disocial, solo una pequeña minoría lo realiza.

Por tanto, se logró identificar a través de estos datos, las competencias que posee el docente para manejar el trastorno disocial escolar en los estudiante en correspondencia con el segundo objetivo específico del estudio. No existe una acción docente generalizada ni mancomunada para impulsar desde el aula de clase estrategias pedagógicas adecuadas para manejar conductas atinentes al comportamiento disocial. Además, se comprobó en el docente equivocación para identificar este tipo de trastorno representado por comportamientos inadecuados que afectan al estudiante. La mayoría muy poco aplica el rol de orientador para brindar al estudiante la asesoría, la ayuda y la consejería para disminuir estos comportamientos en el ambiente escolar.

Como tercer objetivo de la investigación, estos resultados orientan al estudio a establecer el tipo de competencias que necesita el docente para manejar el trastorno disocial escolar en los estudiantes de educación secundaria. Se ha demostrado en la literatura de (Schmal, 2015) que las competencias representa la habilidad y la destreza que posee el profesional para colocar en práctica determinados 
conocimientos. Asimismo señala que las competencias son básicas o genéricas, profesionales también denominadas como especializadas y participativas que cumplen una función transversal en la profesión.

De ahí, como cuarto objetivo específico del estudio, surge la necesidad de diseñar un modelo de acción docente desde la perspectiva humanista como alternativa pedagógica para el manejo del trastorno disocial escolar dirigido a los docentes de educación secundaria de la Unidad Educativa "Alto Barinas Norte", Por lo cual se tomó aspectos relacionados con la descripción de los tipos de comportamiento disóciales que ocurren en estos estudiantes y el conocimiento obtenido de las competencias en el docente. De la misma forma, se incorporaron elementos clave para fortalecer la acción docente mediante una capacitación estructurada por fases que facilitan la adquisición de conocimientos, estrategias que mejoran el desempeño docente y el rol de orientador.

En efecto, una formación con base a competencias para el manejo de comportamientos agresivos, delictivo en el ambiente escolar, así como también, situaciones que están relacionadas con el trastorno disocial escolar. Por ello, la Fig.1 muestra los diversos elementos sobre el modelo de acción docente dirigido a los estudiantes de educación secundaria del plantel, Unidad Educativa "Alto Barinas Norte"

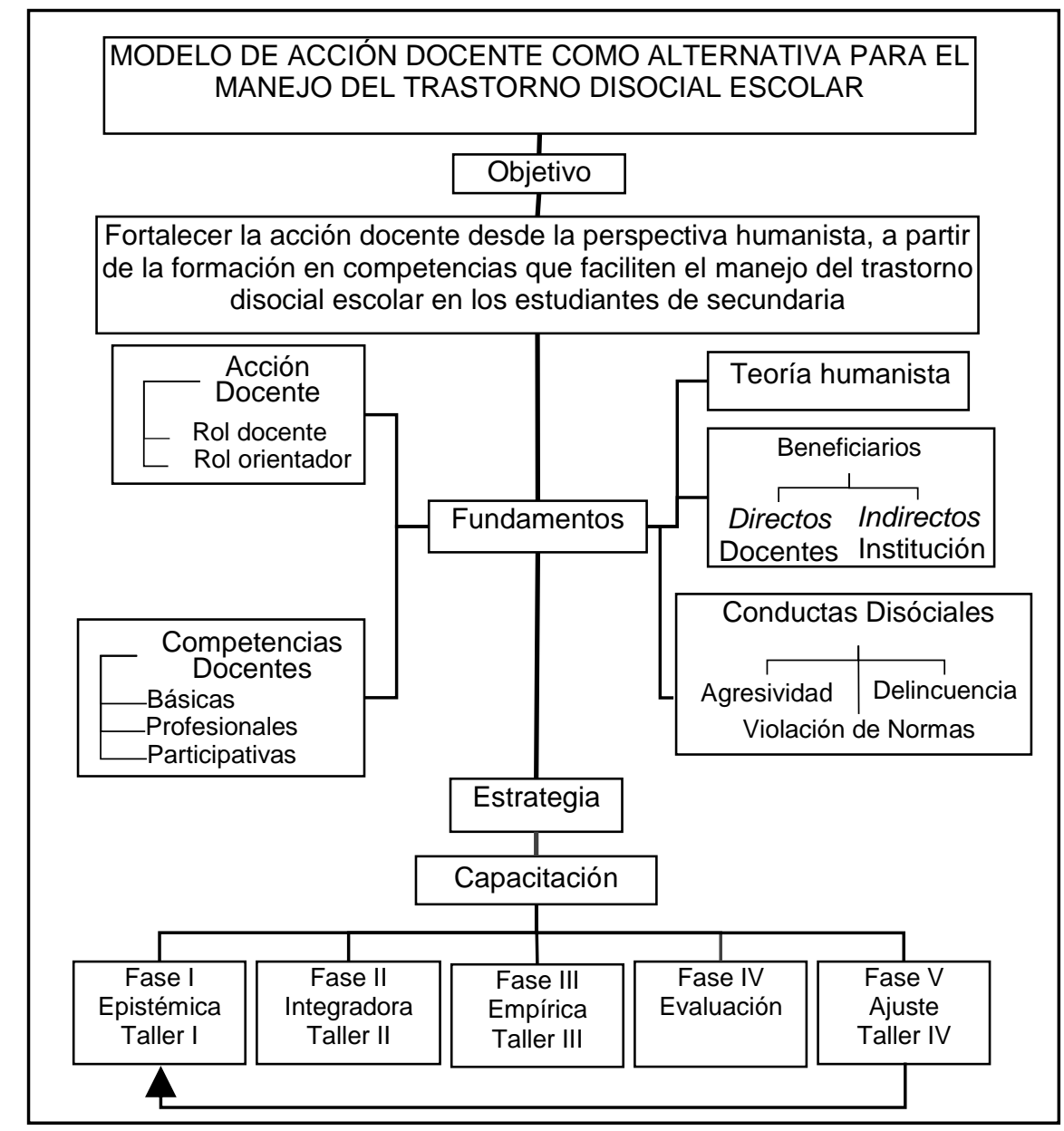

Fig. 1: Modelo de Acción Docente como Alternativa para el Manejo del Trastorno Disocial Escolar

En efecto, la Fig. 1: Modelo de Acción Docente como Alternativa para el Manejo del Trastorno Disocial Escolar, es un esquema epistemológico que representa la realidad objeto de estudio, cuyos componentes educativo están relacionado con el hecho y los actores vinculados a la problemática, facilita la compresión de las características vinculadas con la acción docente tales como: competencias, ejercicio de roles y estrategias aplicadas. Así mismo, refleja la idea del investigador enmarcada en la acción docente como medio para impulsar el proceso educativo y el desarrollo integral del estudiante.

En tal sentido, el modelo tiene como eje de acción, el desarrollo de talleres de capacitación docente desde la perspectiva humanista, a fin de brindar experiencias cognitivas, afectivas y procedimentales que contribuyan a desarrollar competencias para el manejo de conductas relacionadas con el trastorno disocial escolar, en los estudiantes de Educación Secundaria de la Unidad Educativa "Alto Barinas Norte". Ofrece como herramienta viable que beneficiará directamente a los docentes de la institución que desempeñen una práctica pedagógica humanista basada en competencias para alcanzar una educación de calidad. 


\section{DISCUSIÓN FINAL}

En cuanto al primer objetivo específico, referido a la descripción de los tipos de comportamiento disóciales que ocurren en los estudiantes de educación secundaria de la Unidad Educativa "Alto Barinas Norte"; se logró observar y registrar para su clasificación, un conjunto de manifestaciones conductuales caracterizados por la presencia de la agresión verbal y física, delincuencia, burlas e incumplimiento de las normas de convivencia, que en conjunto afectaban el bienestar estudiantil. Estos hechos fueron identificados como evidencias de trastorno disocial, frente a los cuales los docentes no actuaban de manera proactiva, siendo común que obviaran lo ocurrido o adoptasen una actitud complaciente, producto del desconocimiento de estrategias para actuar en tales situaciones o no contar con competencias para ello (González, et al., 2015).

Con relación al segundo objetivo específico, referido a identificar las competencias en el docentes para el manejo del trastorno disocial escolar en los estudiantes de educación secundaria, los resultados demostraron que la mayoría de los docentes, no posee los conocimientos básicos sobre el rol de orientador, aspecto de la praxis docente determinante para proporcionar ayuda al estudiante en su proceso de crecimiento personal, (Torres, 2016). La función orientadora, según (Fernandes et al., 2016), debe formar parte de las competencias en el docente para proporcionar asistencia y asesoría al estudiante, con la finalidad de prevenir comportamientos disociales que puedan afectar la salud y el cumplimiento de las normas de convivencia escolar. Por tal motivo, (Schmal, 2015) señala que en la medida que los docentes fortalezcan las competencias para el desempeño del rol de orientador, se contribuirá a la prevención de comportamientos relacionados con el trastorno disocial escolar.

Seguidamente, el tercer objetivo específico concerniente a establecer el tipo de competencias docentes para el manejo del trastorno disocial escolar en los estudiantes de educación secundaria, los docentes carecen de las competencias básicas, para manejar los comportamientos disociales para proporcionar la orientación necesaria a fin de prevenir consecuencias y situaciones relacionadas con el mismo. Estas competencias, (Marinho y Almeida, 2016), consisten en las habilidades y destrezas que posee el docente para colocar en práctica sus conocimientos a fin de reconocer el comportamiento disocial, solucionar conflictos y prevenir disrupciones que se puedan presentar en el aula de clase. Al respecto, (Bustamante et al., 2016) expresan, las competencias representan un carácter holístico e integrador, como base fundamental para desarrollar la aplicabilidad de las destrezas y los conocimientos para manejar el comportamiento disocial. Con referencia a (Schmal, 2015) las competencias, básicas, profesionales, participativas del docente, son fundamentales en el proceso de formación integral del educando, además coadyuvan al logro de los objetivos de aprendizaje, la toma de conciencia de las dificultades que pueda presentar el estudiante en el ambiente escolar, con la finalidad de prevenir el comportamiento disocial dentro en el ambiente escolar.

Finalmente, el cuarto objetivo específico orientado hacia el diseño del modelo de acción docente desde la perspectiva humanista como alternativa pedagógica para el manejo del trastorno disocial escolar, se tomó en cuenta elementos pertinente que determina con precisión las condiciones reales del objeto de estudio. Este modelo busca desarrollar en el docente las competencias básicas, profesionales y participativas en beneficio del proceso de la enseñanza aprendizaje del estudiante de educación secundaria; ya que si un docente no cuenta con competencias para enfrentar conductas vinculadas a trastornos disociales, el desenvolvimiento de las actividades académicas y el proceso de desarrollo integrado del estudiante se ven afectados, en el ambiente escolar y familiar (Lima, et al., 2017).

En este mismo sentido, (Oses y Carrasco, 2013) deducen que son pocos los docentes en el plantel que cumplen con el rol de orientador aunque afirman cumplirlo o conocerlo para prevenir escenarios vinculados con la agresividad el delito y el cumplimento de las normas de convivencia escolar, cuando la realidad es que el docente no propicia acciones destinadas atender y comprender las necesidades intrínsecas del estudiante, a fin de prevenir comportamientos agresivos dentro del ambiente escolar. En tal sentido, (Marin y Conchado, 2012) afirma, es necesario que el docente asuma este tipo de rol, a fin de impulsar acciones que facilitan el desarrollo de actividades en beneficio del estudiante, obtener un aprendizaje significativo, mediante actividades deportivas, culturales, recreativas, entre otras, que fortalecen los valores humanistas y el buen comportamiento. De allí que cumplir con este rol permita promover la participación organizada en escenarios proclives al crecimiento personal del estudiante.

\section{CONCLUSIONES}

De acuerdo a los resultados y su discusión se pueden obtener las siguientes conclusiones principales:

1.- El modelo de acción propuesto radica en fortalecer el desempeño docente para prevenir en el aula de clase aquellos comportamientos relacionados con la violencia, el delito, la agresividad y el incumplimiento de las normas de convivencia como se evidenció en los resultados del estudio. Dado que el trastorno 
disocial escolar encierra estos comportamientos que son repetitivos que afectan el bienestar estudiantil. El modelo presentado puede considerarse como ejemplo de referencia para otras instituciones educativas adaptado con otros aspectos pedagógicos.

2.- Este nuevo modelo es fácil para su puesta en práctica, ya que basta con seleccionar las fases para desarrollar los talleres de capacitación a fin de brindar al docente experiencias y procedimentales que contribuyan con el perfeccionamiento de competencias para el manejo de estas conductas vinculadas con el trastorno disocial escolar.

3.- Los resultados que se pueden esperar con la ejecución del nuevo modelo de acción docente, son comparables con otros modelos pedagógicos que beneficiará directamente a los estudiantes y docentes de la institución. La metodología que presenta este modelo se encuentra fundamentado en la teoría humanista, lo que permite desarrollar una práctica pedagógica diferente que garantiza la formación de valores en el estudiante para alcanzar una educación de calidad.

\section{REFERENCIAS}

Aarón, M.A., Choles, P.E., y Solano, A.D. Representación del Proceso Formativo de una institución Etnoeducativa a través de la Técnica del Modelo Gráfico y Descriptivo usando Diagramas de influencia y de Forrester, doi: 10.4067/S0718-07642016000300008, Información Tecnológica, 27(3), 81-92 (2016)

Acevedo, D., Cavadia, S., y Alvis, A. Estilos de aprendizaje de los estudiantes de la Facultad de Ingeniería de la Universidad de Cartagena (Colombia), doi: 10.4067/S0718-50062015000400003, Formación Universitaria, 8(4), 15-22 (2015)

Almeida Filho, N. D. For a general theory of health: preliminary epistemological and anthropological notes, doi: 10.1590/S0102-311X2001000400002, Cadernos de Saúde Pública, 17(4), 753-770 (2001)

Aznar, I., y Laiton, I. Desarrollo de Habilidades Básicas de Pensamiento Crítico en el Contexto de la Enseñanza de la Física Universitaria, doi: 10.4067/S0718-50062017000100008, Formación Universitaria, 10(1), 71-78 (2017)

Barrera, M. Modelos Epistémicos en Educación e Investigación, 4aㅗ Ed., 23-42, Sypal, Quirón Ediciones, Caracas, Venezuela (2012)

Bastos, J. L., Duquia, R. P., González-Chica, D. A., Mesa, J. M., y Bonamigo, R. R. Field work I: selecting the instrument for data collection, doi: 10.1590/abd1806-4841.20143884, Anais brasileiros de dermatologia, 89(6), 918-923 (2014)

Blašková, M., Blaško, R., Jankalová, M., y Jankal, R. Key personality competences of university teacher: comparison of requirements defined by teachers and/versus defined by students, doi: 10.1016/j.sbspro.2013.12.731, Procedia-Social and Behavioral Sciences, 114, 466-475 (2014)

Bontempi Jr, B., y Pietri, E.D. Educative processes and the formation of individuals today, doi: 10.1590/s151797022017430200, Educação e Pesquisa, 43(2), 301-309 (2017)

Bryan, V. D., Lindo, J., Anderson-Johnson, P., y Weaver, S. Using Carl Rogers' Person-Centered Model to Explain Interpersonal Relationships at a School of Nursing, doi: 10.1016/j.profnurs.2014.07.003, Journal of Professional Nursing, 31(2), 141-148 (2015)

Bustamante, M. A., Grandón, M. L., Lapo, M., y Oyarzún, C.H. Inteligibilidad de la Docencia de Pregrado: de la Enseñanza por Objetivos al Desarrollo de Competencias, doi: 10.4067/S0718-50062016000500002, Formación Universitaria, 9(5), 03-14) (2016)

Carvalho, R.G., y Novo, R. F. The relationship between structural dimensions of personality and school life in adolescence, doi: 10.1590/1678-7153.201427218, Psicologia: Reflexão e Crítica, 27(2), 368-376 (2014)

Cascaes da Silva, F., Gonçalves, E., Valdivia Arancibia, B.A., Bento, G. G., Silva Castro, T.L.D., Hernández, S., y Silva, R. D. Estimadores de consistencia interna en las investigaciones en salud: el uso del coeficiente alfa, https://goo.gl/FGNEGJ, Rev. Peruana de medicina experimental y salud pública, ISSN1726-464232(1), 129-138 (2015)

Cid, J.E., Cuadra, L.P., Cuevas, S.H., y Villalobos, A. A. Articulación Educación y Trabajo: Un estudio desde la Mirada de los Docentes de la Educación Técnica Regional Chilena y sus Necesidades de Perfeccionamiento Pedagógico, doi: 10.4067/S0718-07642017000100004, Información Tecnológica, 28(1), 25-34 (2017)

Contreras, D.L.M. Orientación educativa: práctica de intervención permanente en los centros escolares, https://goo.gl/DJW8wt, E-ISSN: 1696-2095, Rev. Redalyc, 7(19), 1449- 1460 (2009)

Dutra, E.M.D.S. Rogers and Heidegger: Is a gathering for a new view of the self-possible? doi: 10.1590/198202752016000300005, Estudos de Psicologia (Campinas), 33(3), 413-423 (2016)

Faleiros, F., Käppler, C., Pontes, F. A. R., Silva, S. S. D. C., Goes, F. D. S. N. D., y Cucick, C. D. Use of virtual questionnaire and dissemination as a data collection strategy in scientific studies, doi: 10.1590/010407072016003880014, Texto y Contexto-Enfermagem, 25(4) (2016)

Fernandes, D.J., Sotolongo, M., y Martínez, C.C. La Evaluación del Desempeño por Competencias: Percepciones de Docentes y Estudiantes en la Educación Superior, doi: 10.4067/S0718-50062016000500003, Formación Universitaria, 9(5), 15-24 (2016) 
Flores, F. J., Peinado, J. E., Ornelas, M., y López, L. Composición Factorial de una Escala Autoeficacia en Conductas de Cuidado de la Salud en Estudiantes de Ingeniería, doi: 10.4067/S0718-50062012000300006, Formación Universitaria, 5(3), 43-54. (2012)

García Valdés, M., y Suárez Marín, M. El método Delphi para la consulta a expertos en la investigación científica, https://goo.gl/74QNcK, ISSN: 1561-3127, 253-267, 39(2) 253-267 (2013)

González, A., Paoloni, P. V., Donolo, D., y Rinaudo, C. Behavioral engagement and disaffection in school activities: exploring a model of motivational facilitators and performance outcomes, doi:10.6018/analesps.32.1.176981, Anales de Psicología, 31(3), 869-878 (2015)

Hernández, R. Fernández, C. y Baptista, P. Metodología de la Investigación. 6ª Ed., 270-299, Mc Graw Hill, DF. México (2014)

Jorm, A.F. Using the Delphi expert consensus method in mental health research. doi: 10.1177/0004867415600891, Australian \& New Zealand Journal of Psychiatry, 49(10), 887-897 (2015)

Lapponi, S. F. Teachers' emotions: affection and anger as resources for disciplining, doi: 10.1590/s15179702201610149719, Educação e Pesquisa, (AHEAD), 0-0 (2016)

Lima, R. M., Dinis-Carvalho, J., Sousa, R. M., Arezes, P., y Mesquita, D. Development of competences while solving real industrial interdisciplinary problems: a successful cooperation with industry, doi;10.1590/0103-6513.230016, Production, 27(SPE) (2017)

Marin-Garcia, J.A., y Conchado-Peiró, A. Influencia de los Perfiles Docentes en el Uso y Preferencia de Dinámicas de Trabajo en Grupo con Alumnos Universitarios, doi: 10.4067/S0718-50062012000500002, Formación Universitaria, 5(5), 3-14 (2012)

Marinho-Araujo, C.M., y Almeida, L.S. Approach to competences, human development and higher education, doi: 10.1590/0102-3772e32ne212, Psicologia: Teoria e Pesquisa, 32(SPE) (2016)

Ornelas, M., Blanco, H., Gastélum, G., y Chávez, A. Autoeficacia percibida en la conducta académica de estudiantes universitarias, doi: 10.4067/S0718-50062012000200003, Formación Universitaria, 5 (2),17-26 (2012)

Oses, S. E., y Carrasco, L. E. Módulos Alternativos en la Enseñanza de las Ciencias: Estrategia Didáctica Orientada al Logro de Aprendizajes Significativos, doi: 10.4067/S0718-50062013000300006, Formación Universitaria, 6(3), 39-52 (2013)

Peña-Olvera, F.D.L., y Palacios-Cruz, L. Trastornos de la conducta disruptiva en la infancia y la adolescencia: diagnóstico y tratamiento, https://goo.gl/7J2ADe, ISSN: 0185-3325, Rev. Salud mental, 34(5), 421-427 (2011)

Pérez Solís, M. ¿Puede un psicopedagogo desempeñar el rol del psicólogo educativo? Profesionalización frente al intrusismo profesional, https://goo.gl/y1QqCQ, Estud. psicol. Campinas, 28(1), 3-13 (2011)

Pozo Iribarría, J., y González Izquierdo, A. Encuesta sobre consumo de drogas en jóvenes de la Rioja, 2008, ISBN: 97884-8125-330-6, consejería de salud, dirección general de Salud Pública y Consumo. Servicio de Drogodependencias (2009)

Rosa, G., Riberas, G., Navarro-Segura, L., y Vilar, J. El coaching como herramienta de trabajo de la competencia emocional en la formación de estudiantes de educación social y trabajo social de la Universidad Ramón Llull, España, doi.org/10.4067/S0718-50062015000500009, Formación Universitaria, 8(5), 77-90 (2015)

Schmal, R. Evolución de un Programa de Formación en Competencias Genéricas, Formación Universitaria, doi: 10.4067/S0718-50062015000600012, Formación Universitaria, 8(6), 95-106 (2015)

Todorescu, L. L., Popescu-Mitroi, M. M., y Greculescu, A. Students' Views on the Teacher as An Information Provider, doi: 10.1016/j.sbspro.2015.07.121, Procedia-Social and Behavioral Sciences, 197, 184-189 (2015)

Torres, J.D., Acevedo, D., y Montero, P. M. Proyectos de Aula Semestrales como Estrategia Pedagógica para la Formación en Ingeniería, doi: 10.4067/S0718-50062016000300004, Formación Universitaria, 9(3), 23-30 (2016)

Urazán-Torres, G.R., Puche-Cabrera, M.J., Caballero-Forero, M., y Rey-Anacona, C.A. Cognitive and Executive Functions in Colombian School Children with Conduct Disorder: Sex Differences, https://goo.gl/aobx4S, Rev. Colombiana de Psiquiatría, 42(4), 324-332 (2013)

Vera, Pavez, J., Ezpeleta, L., y Granero, R. Universidad Autónoma de Barcelona, Conducta antisocial: asociación con psicopatología en niños y adolescentes de México http://www.tdx.cat/handle/10803/98471, ISBN: 9788449033292 , Departamento de Psicología Clínica de la Salud, 45, s124-s131 (2013)

Vieira, E.M., y Pinheiro, F.P.H.A. Person centered psychotherapy: an encounter with oneself or a confrontation with the other? doi: 10.1590/S0103-166X2013000200009, Estudos de Psicologia (Campinas), 30(2), 231-238 (2013)

Zalyaeva, E. O., y Solodkova, I. M. Teacher-student collaboration: Institute of economics and finance Kazan federal university approach, doi:10.1016/j.sbspro.2014.09.271, Procedia-Social and Behavioral Sciences, 152, 1039-1044 (2014) 